\section{OTH-03 IMPACT OF DIRECT ACCESS IBD PHYSICIAN DELIVERED SIGMOIDOSCOPY FROM ACUTE IBD FLARE LINE CALL SERVICE}

${ }^{1}$ Richard Harris, ${ }^{1}$ Martin McDonnell, ${ }^{1}$ Ms Louise Downey, ${ }^{1}$ Ms Anne Sanderson, ${ }^{1} \mathrm{Ms}$ Marion Bettey, ${ }^{1}$ Ms Maria Rubio-Padillo, ${ }^{2}$ Ms Florina Borca, ${ }^{2}$ Hang Phan, ${ }^{1}$ Richard Felwick, ${ }^{1}$ Fraser Cummings, ${ }^{1}$ Markus Gwiggner*. ${ }^{1}$ University Hospital Southampton, UK ; ${ }^{2}$ University of Southampton NIHR Biomedical Research Facility, UK

\subsection{6/gutjnl-2019-BSGAbstracts.422}

Introduction Inflammatory bowel disease (IBD) patients calling our specialist nurse led IBD flare line with worsening symptoms were traditionally seen in outpatient clinics before being referred for endoscopy to evaluate disease activity. Non-IBD endoscopists often deferred treatment decisions until subsequent IBD outpatient appointments (OPA), leading to delays in treatment and pressure on IBD OPA workload.

Methods In January 2016 "flare slots" on dedicated IBD endoscopy lists were introduced. Patients with flare symptoms submitted stool for enteric pathogen exclusion and those subsequently deemed suitable were directly booked to a flare slot on an IBD endoscopy list, if available. Anonymised data was systematically extracted from clinical systems for the first 6 months of 2016, 2017 and 2018 regarding endoscopy outcomes leading to change in treatment and number of OPA in the respective years. Change in treatment was defined as start/ stop of medication, urgent investigation to guide treatment or admission to hospital.

\section{Outpatient appointments}

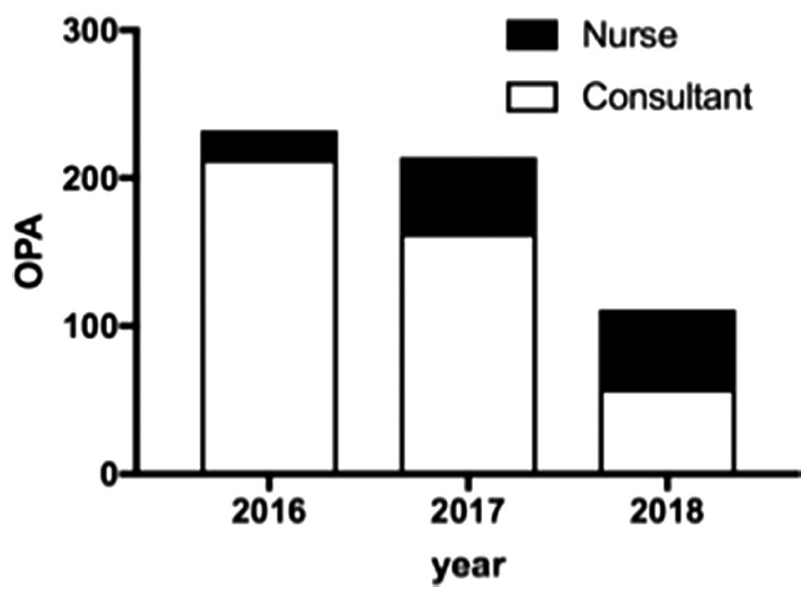

Abstract OTH-03 Figure 1 demonstrates the reduction of IBD OPA by $52.2 \%$ while increasing IBD nurse led OPA by $47.7 \%$.

Results For the first six months of 2016, 2017 and 2018, data identified 90, 92 and 92 IBD flare line-triggered flexible sigmoidoscopies respectively. Overall $74.6 \%$ had ulcerative colitis and the remainder Crohn's disease $(22.3 \%)$ or IBDU (1.3\%). In 2016, 63.3\% of procedures were performed by IBD physicians, which increased to $77.2 \%$ and $79.3 \%$ in 2017 and 2018 respectively. IBD endoscopists initiated change in treatment following their procedures in 70.2\% (2016), 74.7\% (2017) and $84.9 \%$ (2018) of cases, compared to $9.1 \%, 14.3 \%$ and $5.3 \%$ of non-IBD endoscopist procedures over the same period. Total gastroenterology outpatient appointments within the year in this population were 232 (2016), 214 (2017) and 111 (2018); a 52.2\% decrease from 2016 to 2018. Nurse-led outpatient appointments following IBD flare flexible sigmoidoscopy increased from 19 in 2016 to 51 in 2017 and 53 in 2018, when they constituted $47 \%$ of OPA in this group. This represented a $72.8 \%$ (155 OPA) reduction in consultant appointments from 2016 to 2018 (Figure1).

Conclusion Changing the model of service delivery for acute IBD flares by combining IBD physician delivered endoscopy with proactive management decisions resulted in a change of management in $84.9 \%$ of patients. This reduced overall IBD OPA by $52.2 \%$, while increasing nurse led follow up clinics in this cohort to $47 \%$ leading to a total $72.2 \%$ reduction in consultant OPA.

\section{OTH-04 DEVELOPMENT OF A LICENCED FAECAL MICROBIOTA TRANSPLANT SERVICE FOR PATIENT TREATMENT IN THE NHS}

\begin{abstract}
1,3Victoria L McCune, ${ }^{2,3}$ Mohammed Nabil Quraishi* ${ }^{3}$ Susan Manzoor, ${ }^{3}$ Ms Sahida Shabir ${ }^{3}$ Peter M Hawkey, ${ }^{2,3}$ Tariq H Iqbal. ${ }^{1}$ Public Health England Public Health Laboratory Birmingham, Birmingham, UK; ${ }^{2}$ Department of Gastroenterology, University Hospitals Birmingham NHS Foundation Trust, Birmingham,UK; ${ }^{3}$ Microbiome Treatment Centre, Institute of Microbiology and Infection, University of Birmingham, Birmingham, UK
\end{abstract}

\subsection{6/gutjnl-2019-BSGAbstracts.423}

Introduction Faecal Microbiota Transplant (FMT) is an efficacious treatment for recurrent Clostridium difficile infection (CDI) and has shown encouraging signals for inflammatory bowel disease. Access to FMT services in the NHS has been limited by the regulation of FMT as a medicinal product in the UK. To enable inter-institution supply, FMT manufacturers are required to hold a Specials licence from The Medicines and Healthcare products Regulatory Agency.

Methods In 2017 the first licenced FMT service in UK was created at the University of Birmingham Microbiome Treatment Centre. Policies and procedures were developed using the Rules and Guidance for Pharmaceutical Manufacturers and Distributors (Orange Guide), which were used and maintained within a quality management system. The frozen FMT bank utilises anonymous unrelated healthy donors and provides FMT on a named patient basis for the treatment of CDI and supplies clinical trials via an investigational medicinal product (IMP) licence.

Results Since August 2018, 53 faecal microbiota transplants have been supplied for recurrent and refractory CDI to 21 NHS Trusts across England and Wales. Twelve of these Trusts did not have access or perform FMT prior to the introduction of this service. In 98\% (52/53) of cases FMT was delivered either within 48 hours or on the day of request if $>48$ hours from request. Clinical response to treatment was observed in $76 \%(16 / 21)$ of patients, with $88 \%(14 / 16)$ of these patients remaining symptom free at 90 days. In 2018 the service became the sole supplier of FMT under the 201-019 NHS Innovation and Technology Tariff. Under the IMP licence the service has successfully supplied over 360 faecal microbiota transplants for the pilot stage of a multi-centre trial of FMT in UC (STOP-Colitis).

Conclusions Development of a licenced FMT facility at the University of Birmingham has improved equality of access to this novel treatment across the NHS. FMT has been provided within a zero cost model for CDI and is available generally within 48 hours of request. Patients with CDI benefit from improved care from this service, which limits morbidity and mortality from this severe infection. Supply via an IMP licence 
has enabled the required FMT infrastructure to be developed to support prospective FMT research in the UK.

\section{OTH-05 A REVIEW FOLLOWING A DR FOSTER'S REPORT OF APPARENTLY HIGH GASTROINTESTINAL HAEMORRHAGE MORTALITY}

'Duncan Napier*, 'Kate Hale, ${ }^{2}$ Hazel Woodland, ${ }^{1}$ Richard Makins. ${ }^{1}$ Cheltenham General Hospital, Cheltenham, UK; ${ }^{2}$ Bristol Royal Infirmary, Bristol, UK

\subsection{6/gutjnl-2019-BSGAbstracts.424}

Introduction Dr Foster publishes annual mortality rates for every hospital trust and compares these across the NHS. Mortality rate indicators are mechanisms by which the NHS and hospital trusts can identify potentially poorly performing services to facilitate investigation and change. Data is collected from Hospital Episode Statistics (HES) which relies on accurate coding from the first two episodes of care within an hospital and linked to Office for National Statistics data. Dr Foster produced a report looking at mortality at Gloucestershire Hospitals NHS Foundation Trust between October 2015 and September 2016, whereby mortality from gastrointestinal haemorrhage $(\mathrm{GH})$ was statistically higher than expected. This review sought to evaluate the accuracy of the Dr Foster report.

Methods Data submitted to HES via Secondary Uses Services, used in the creation of the Dr Foster report was analysed. Hospital records were reviewed for each patient for the period leading up to their death. Admission diagnosis was recorded as determined by clinical documentation from the first two or three episodes of care. Cause of death was determined by review of death certificates or contact with the regional Coroner's office. Subsequently, all patients with a coding diagnosis relevant to $\mathrm{GH}$ during the study period were listed and notes were reviewed of those who died within or up to 30 days after the study period. The confirmed diagnosis and cause of death were compared with the coding diagnosis from admission documentation.

Results 49 patients were reported to have died from GH compared to an expected number of 35.5 according to the $\mathrm{Dr}$ Foster report, 1 of which was a duplicate. 43 notes were reviewed (5 incomplete or unavailable) and only 32 of these had a differential diagnosis of $\mathrm{GH}$ within the first 3 clinical episodes. However, only 12 of these ultimately had GH listed as a cause of death on the patient's death certificate. On subsequent analysis of all 61 patients who died with a code suggestive of $\mathrm{GH}$ during their admission, 39 had a differential diagnosis of $\mathrm{GH}$ within the first three episodes.

Conclusions Dr Foster relies on accurate coding and a correct initial diagnosis to provide robust data on hospital mortality. Our study shows that coding within the study was often inaccurate but more importantly that the initial diagnosis (within the first few clinical episodes) often did not reflect the confirmed diagnosis after specialist review and investigations were completed. In addition, patients' death certificates recorded a cause of death unrelated to $\mathrm{GH}$ in many cases, the commonest being bronchopneumonia. Admission diagnosis often fails to reflect the actual admission diagnosis or cause of death and can result in inaccurate mortality statistics.

\section{OTH-06 OUTCOMES FROM THE FIRST FORMALISED NATIONAL ENDOSCOPY SERVICE IN SIERRA LEONE}

${ }^{1}$ DE Thomas-Macauley ${ }^{*},{ }^{1} S$ Conteh, ${ }^{1} \mathrm{~F}$ Ngongo, ${ }^{2} \mathrm{~J}$ Hancock, ${ }^{3} \mathrm{C}$ Mountford, ${ }^{3} \mathrm{DL}$ Nylander, ${ }^{2} \mathrm{CW}$ Wells, ${ }^{2} \mathrm{R}$ Bevan . ${ }^{1}$ Choithram Memorial Hospital, Freetown, Sierra Leone; ${ }^{2}$ North Tees and Hartlepool NHS Foundation Trust, UK; ${ }^{3}$ Newcastle Upon Tyne Hospitals NHS Foundation Trust, UK

\subsection{6/gutjnl-2019-BSGAbstracts.425}

Introduction Sierra Leone is a developing country in Africa, with a population of 7 million. Prior to 2016 there was no recognised endoscopy service in the country, although one hospital in the capital Freetown (pop. 1 million) owned an endoscopy stack. Medical services are rudimentary, but with a small group of motivated doctors and a grant from the BSG, a training programme was developed to support the provision of endoscopy.

Here we describe the outcomes from the service since it's inception in December 2016.

Methods 3 endoscopists have been trained in OGD, and are supported remotely by 5 clinicians in the UK via Whatsapp. An annual training visit has been undertaken to consolidate skills. Weekly endoscopy lists are delivered in Choithram Hospital, with reports generated on ADAM endoscopy reporting software. An Excel spreadsheet database of all procedures is maintained by the endoscopists.

The database was interrogated for procedures since the start of the service up to the end of January 2019. Data were collected on gender, age, intubation of the second part of the duodenum, length of procedure, CLO test results and endoscopic findings.

Results 289 procedures have been performed. 56\% were male, median age 43 years (range 1-8). The number of procedures increased significantly in the second year of the service. The most frequent referral reason is dyspepsia (62\%)

Overall results are shown in table 1.

Pathology detection rates have been similar in each year of the programme. 18 cancers were identified, 25 cases with ulcers, and 21 cases with evidence of portal hypertensive changes (oesophageal/gastric varices, or portal gastropathy).

\begin{tabular}{|c|c|c|c|}
\hline & $\begin{array}{l}\text { December } 2016 \text { - end } \\
\text { December } 2017\end{array}$ & $\begin{array}{l}\text { January } 2018 \text { - end } \\
\text { January } 2019\end{array}$ & Total \\
\hline Procedures & 74 & 215 & 289 \\
\hline CLO test performed* & 3 & 163 & 166 \\
\hline +ve result (\%) & $1(33)$ & $112(69)$ & $113(68)$ \\
\hline D2 reached $(\%)^{*}$ & $64 / 73(88)$ & 192/202 (95) & $\begin{array}{l}256 / 275 \\
(93)\end{array}$ \\
\hline $\begin{array}{l}\text { Mean procedure length, } \\
\text { mins }(S D)^{*}\end{array}$ & $31(16.4)$ & $19(8.5)$ & $\begin{array}{l}22 \\
(12.0)\end{array}$ \\
\hline Pathology detected (\%)* & $55 / 72(76)$ & 155/203 (75) & $\begin{array}{l}210 / 275 \\
(76)\end{array}$ \\
\hline
\end{tabular}

*complete data not available for all procedures - denominator given in each case

Discussion Sierra Leone now has a functioning upper GI endoscopy service. This service is providing high quality procedures, with overall D2 intubation rate meeting the JAG requirement of 95\%. Pathology detection rates are high, possibly due to current practice of only those with the most severe symptoms being referred. Clinically important lesions (ulcers, cancers and 